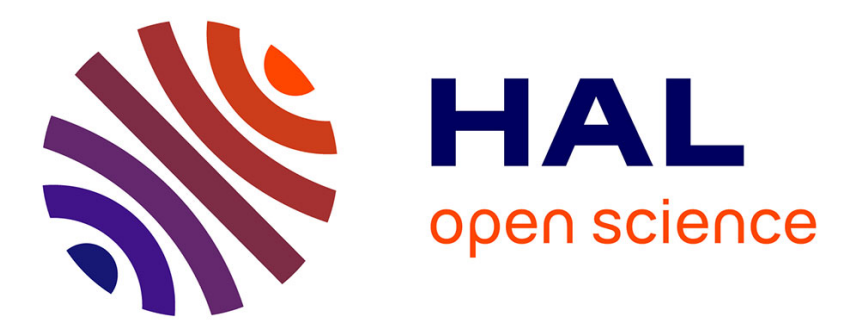

\title{
Backward Reachability Analysis of Colored Petri Nets
}

Mohamed Bouali, Jérôme Rocheteau, Pavol Barger

\section{To cite this version:}

Mohamed Bouali, Jérôme Rocheteau, Pavol Barger. Backward Reachability Analysis of Colored Petri Nets. The European Safety and Reliability Conference (ESREL'09), Sep 2009, Prague, Czech Republic. pp.1975-1981. hal-00447509

\section{HAL Id: hal-00447509 \\ https://hal.science/hal-00447509}

Submitted on 15 Jan 2010

HAL is a multi-disciplinary open access archive for the deposit and dissemination of scientific research documents, whether they are published or not. The documents may come from teaching and research institutions in France or abroad, or from public or private research centers.
L'archive ouverte pluridisciplinaire HAL, est destinée au dépôt et à la diffusion de documents scientifiques de niveau recherche, publiés ou non, émanant des établissements d'enseignement et de recherche français ou étrangers, des laboratoires publics ou privés. 


\title{
Application of linear logic to Backward Reachability Analysis of Colored Petri Nets
}

\author{
Mohamed BOUALI, Jérôme ROCHETEAU \& Pavol BARGER \\ Heudiasyc Laboratory, CNRS UMR 6599 \\ Université de Technologie de Compiègne, France \\ Tel: +33344234423 \\ Fax : +33344234477 \\ \{mohamed.bouali, jerome.rocheteau, pavol.barger\}@hds.utc.fr
}

\begin{abstract}
This paper deals with a formal method for the study of the backward reachability analysis applied on Colored Petri Nets (CPN). The proposed method proceeds in two steps : 1) it translates CPN to terms of the Multiplicative Intuitionistic Linear Logic (MILL); 2) it proves sequents by constructing proof trees. The translation from CPN to MILL must respect some properties such as the semantic associated to tokens. That is why, the First-Order MILL (MILL1) is used for translation. The reachability between two markings, the initial marking and the final marking, is expressed by a sequent which can be proven (if the initial marking is backward-reachable from the final one) using first-order terms unification and/or marking enhancement.
\end{abstract}

\section{Introduction}

System dependability is an important research issue especially if applied to critical domains. Systems are verified through tools that check the compliance of their properties with the design specifications. Formal methods provide an interesting way to study and develop verification tools thanks to their precise characterization of the modelled system. It is in this context that the presented work applies a formal method approach to system modelling and analysis.

Petri Nets (PN) (Petri 1962), and especially, Colored Petri Nets(CPN) (Jensen and Rozenberg 1991), are a powerful and recognized modelling tool. They are endowed with a big expressiveness and allow to represent the two aspects of a system : static thanks to the PN structure and dynamic thanks to the token evolution. The PN analysis can be done in several manners like exhaustive reachable state space enumeration or Monte Carlo simulation (Metropolis and Ulam 1949). These methods allow to study request/action effects on the model behavior, but they suffer from drawbacks such as combinatorial explosion and results correctness (some results are bound with a potentially known error range). To avoid these drawbacks, we propose to use the structural analysis using directly the model itself. In addition, this work is particularly interested in the diagnosis and the fault driven analysis. That means, by knowing a particu- lar final state (which represents a failure state), the sources of this state can be found. In such analysis, all possible initial configurations must be studied to find those leading to the considered failure state. This is why, it is interesting to study the reachability between two markings studied under two dual manners (Khalfaoui 2003): the forward reachability and the backward reachability. The first case consists in building state successors one by one, starting with the initial marking $M_{0}$ and ending with the final marking $M_{f}$. Thus, considering $M_{0}$ as the present state, $M_{f}$ is considered as the future state. In the backward reachability, $M_{f}$ constitutes the present state and $M_{0}$ is regarded as the past state. The general idea is to build, from the present state, the predecessor states until the reach of the past state which is logically the source of the present marking.

To perform the backward reachability analysis over CPN, (M.Bouali, P.Barger, and W.Schon 2009a; M.Bouali, P.Barger, and W.Schon 2009b) developed a method based on inverse Colored Petri Net (inverse CPN) which results from transformations applied on the original CPN. Knowing that, in the case of ordinary Petri Nets, the linear logic offers another more formal method to study reachability between to states, the idea is to generalize this method to CPN and especially for inverse CPN. The equivalence between structural analysis and linear logic (applied to CPN) 
can be obtained by performing appropriate translation from CPN to linear logic. The linear logic formalism is very interesting because it can constitute a formal proof for the structural backward reachability.

CPNs model the system structure and its dynamic behavior in the same model. The dynamic behavior is modelled thanks to token evolution. After each transition firing, some tokens are consumed and some other are produced. This notion of production/consumption cannot be expressed in classical logic, that is why the MILL was preferred. On the other hand, unlike Ordinary Petri Nets, token in CPN is of a certain type (color) and belongs to a set of this type (color set) and is transformed by arc expressions. So the translation from CPN to MILL must respect these properties. That is why, the First-Order MILL (MILL1) is used for the translation. CPN Places are expressed in MILL1 by unary relation symbols (Propositional variables) which allow to deal with arc expressions and token values. CPN transitions are expressed in MILL1 by implicative formulas allowing introduction of universal quantifiers. The reachability between the two markings $M_{0}$ (initial marking) and $M_{f}$ (final marking) is expressed by a sequent which can be proven (if $M_{0}$ is backward-reachable from $M_{f}$ ) using first-order terms unification and/or marking enhancement.

This paper is organized as follows : The section 2 gives definitions of Petri Nets and Colored Petri Nets. It also introduces the structural backward reachability in CPN. The section 3 presents the Linear Logic and its relation with Petri Nets. It is followed, in the section 4 , by the details of translation from CPN to MILL1 and the reachability analysis using sequents in linear Logic. The section 5 contains a presentation of the case study. The paper ends by the conclusion and an outline of future perspectives.

\section{Structural Backward Reachability for Colored} Petri Nets

\subsection{Petri Net and Colored Petri Net}

A Petri Net (Petri 1962), called also Place/Transition Net, is a directed bipartite graph defined by the 4tuple $(P, T$, Pre, Post), where: $P$ is a finite set of places, $T$ is a finite set of transitions $(P \cap T=\emptyset)$, Pre is the backward incidence application, Post is the forward incidence application.

The notation of Colored Petri Net (CPN) (Jensen and Rozenberg 1991) introduces the notion of token types, namely tokens are differentiated by colors, which may contain arbitrary data values. Each place has an associated type determining the kind of data that the place may contain. A non-hierarchical CPN is defined by the 9-tuple $(\Sigma, P, T, A, N, C, G, E, I)$ where :

- $\Sigma$ is a finite set on non-empty types,
- $P$ is a finite set of places,

- $T$ is a finite set of transitions,

- $A$ is a finite set of $\operatorname{arcs}$ such that: $P \cap T=P \cap$ $A=T \cap A=\emptyset$,

- $N$ is a node function. It is defined from $A$ into $P \times T \cup T \times P$,

- $C$ is a color function. It is defined from $P$ into $\Sigma$,

- $G$ is a guard function. It is defined from $T$ into expressions such that:

$\forall t \in T:[\operatorname{Type}(G(t))=B \wedge T y p e(\operatorname{Var}(G(t))) \subseteq \Sigma]$,

- $E$ is an arc expression function. It is defined from $A$ into expressions such that:

$\forall a \in A:\left[\operatorname{Type}(E(a))=C(p(a))_{M S} \wedge\right.$

$$
\text { Type }(\operatorname{Var}(E(a))) \subseteq \Sigma]
$$

Where $p(a)$ is the place of $N(a)$,

- $I$ is an initialization function. It is defined from $P$ into closed expressions such that:

$$
\forall p \in P:\left[\operatorname{Type}(I(p))=C(p)_{M S}\right]
$$

\subsection{Colored Petri Net inversion}

Backward reachability in CPNs is a dual concept of the forward reachability. That is, if a marking $M_{f}$ is reachable from $M_{0}, M_{0}$ is said backward reachable from $M_{f}$. Backward-reachability means that $M_{0}$ is a cause or a source of $M_{f}$. To perform the backward reachability analysis, an inverse CPN is used. It is obtained by application of a set of structural transformations on the original CPN. These transformations are directly dependent on the CPN structure. Consequently, we have to define a transformation rule for each structure case studied. Nevertheless, the most common transformations in representative cases are presented bellow.

Basic transformation rule : Tab.1.a shows a trivial case of a CPN inversion: input and output arcs are marked with constants $a, b$. Tab.1.b shows a case where the input arc is marked with variable $x$, the output arc is marked with function $f(x)$ and a guard $G(x)$ is associated to the transition $t$. 
Mixed transformation rule : Tab.1.c shows a mixed case where some input arcs are marked with variables $\{x, y\}$ and other arcs by constants $\{a, b\}$. Output arcs are marked with constants $\{c\}$ and reversible functions $\{f, g\}$. This $\mathrm{CPN}$ inversion is a mix (generalization) of the basic transformation. Each variable is used by one and only one function. In the opposite case, see the Parametric transformation below.

Parametric transformation rule : Some CPN structures can't be reversed to get deterministic markings in the backward reachability. The reason is that the inversion process could be assimilated to mathematic operations whose solutions may be intervals. The CPN inversion, in these cases, is parametric. That means, some additional information, like color sets, are needed. Two cases follow : the first concerns arc expressions which are multivariable equations (Tab.1.d), the second treats input variables which are not associated with output functions (Tab.1.e).

Parallel transformation rule : The term 'parallel' means existence of a shared variable. Tab.1.f shows the case where the same variable is used by more than one function (two functions in this case).

\section{Linear Logic}

Linear logic was introduced by Girard (Girard 1987). Its expressive power is demonstrated by some very natural encodings of computational models such as Petri Nets, counter machines, Turing machines, and others (Lincoln 1992). Linear logic differs from classical logic by introducing the notion of a ressource. Classical logic deals with static propositions where each proposition is either true or false. Because of the static nature of propositions in classical logic, there may be duplicated $[P \Rightarrow(P \wedge P)]$ and/or discarded $[(P \wedge Q) \Rightarrow P]$. Both of these propositions are valid in classical logic for any $P$ and $Q$. In linear logic, these propositions are not valid because no information is available about how the second $P$ is produced (in the first proposition) or where $Q$ is consumed (in the second proposition). The rules of linear logic imply that linear propositions stand for dynamic properties or finite resources.

For example, consider the propositions $\mathrm{E}, \mathrm{C}$, and T, conceived as resources: 1)E: one Euro, 2)C: cup of Coffee, 3)T: cup of Tea. Consider the following axiomatization of a vending machine: 1) $E \Rightarrow C, 2) E \Rightarrow$ $T$. Then in classical logic, the proposition $E \Rightarrow(C \wedge$ $T)$ can be deduced. Which may be read as "With one Euro, I may buy both a cup of coffee and a cup of tea". Although this deduction is valid in classical logic, it is nonsense in the intended interpretation of propositions as resources: two cups of hot drinks cannot be

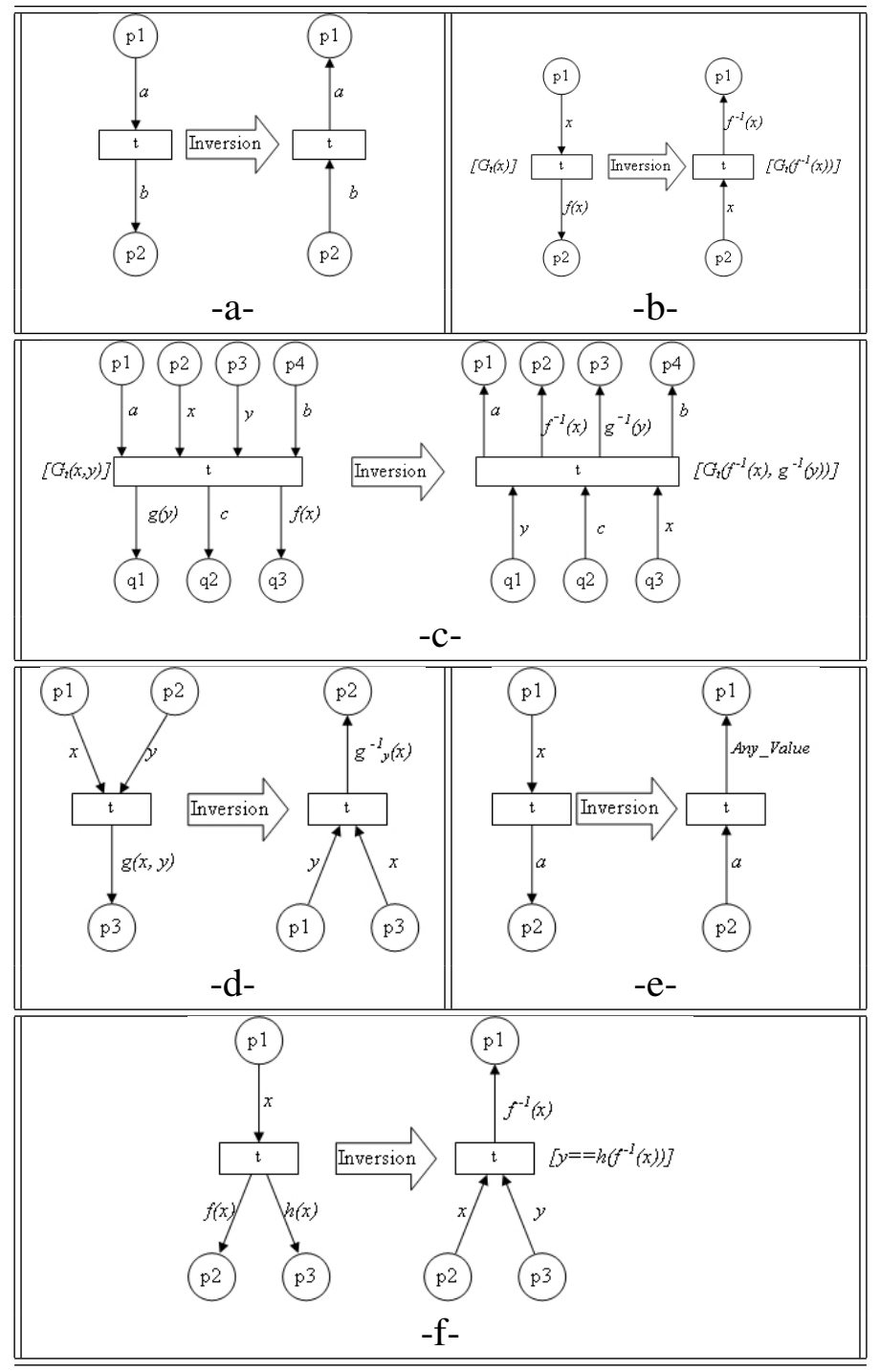

Table 1: CPN transformations for inversion 
bought with one Euro from the described vending machine.

In this work, we only use a fragment of the linear logic related to Petri Nets which is MILL fragment (Multiplicative Intuitionistic Linear Logic). This fragment contains the multiplicative connector TIMES $(\otimes)$ and linear implication connector $(-)$. The TIMES connector traduces the accumulation of resources. The proposition $A \otimes A$ means that two resources $A$ are available. The Linear implication $(-)$ expresses the causality between production and consumption of resources. The proposition $A \multimap B$ means that when the proposition $A$ is consumed, the proposition $B$ is produced. The meta-connector "," (comma) is cumulative (Girard 1987).

The sequent calculus notation, due to Gentzen (G.Gentzen 1969), is composed of two sequences of formulas separated by a turnstile symbol $(\vdash)$. The formula $\Gamma, \Gamma^{\prime} \vdash \Delta, \Delta^{\prime}$ means that the conjonction of $\Gamma$ and $\Gamma^{\prime}$ allows to deduce the disjonction $\Delta$ or $\Delta^{\prime}$. A sequent calculus proof rule consists of a set of hypothesis sequents, written above a horizontal line, and a single conclusion sequent, written below the line, as follow:

$$
\frac{\text { Hypothesis }_{1} \quad \text { Hypothesis }_{2}}{\text { Conclusion }_{\text {Rule }} \text { attribute }}
$$

The goal is to construct a proof tree. Starting from the sequent, and applying step by step some adapted rule, the proof consists on eliminating the connectors. These rules are shown in Fig. 1 where : $A$ is an atom ; $F, G$ and $H$ are formulas ; $\Gamma$ and $\Delta$ are blocs of formulas separated by commas. The attribute indicates whether the rule is applied at left $(L)$, at right $(R)$ or to the whole sequent (empty attribute).

$$
\begin{aligned}
& \frac{\Gamma \vdash F \quad \Delta, F \vdash H}{\Gamma, \Delta \vdash H} C u t \\
& \frac{\Gamma, F, G, \Delta \vdash H}{\Gamma, G, F, \Delta \vdash H} \text { Exchange } \\
& \frac{\Gamma \vdash F \quad \Delta, G \vdash H}{\Gamma, \Delta, F \multimap G \vdash H} \multimap L \\
& \frac{\Gamma, \Delta, F \vdash G}{\Gamma, \Delta \vdash F \multimap G} \multimap_{R} \\
& \frac{\Gamma, F, G \vdash H}{\Gamma, F \otimes G \vdash H} \otimes_{L} \quad \frac{\Gamma \vdash F \quad \Delta \vdash G}{\Gamma, \Delta \vdash F \otimes G} \otimes_{R}
\end{aligned}
$$

Figure 1: Sequent calculus rules of the MILL fragment

The interest of linear logic is that it provides, for example, a natural and simple coding of Petri Net reachability (Lincoln 1992). Based on the sequent calculus, linear logic helps to get a necessary and sufficient condition of reachability from one marking to another, thanks to the equivalence between the reachability in a Petri Net and the provability of the corresponding sequent (F.Girault 1997). Moreover linear logic gives the partial firing order of the different transitions to reach a final marking $M_{f}$ from an initial one $M_{0}$ (H.Demmou, S.Khalfaoui, E.Guilhem, and R.Valette 2004).

To translate a Petri Net to linear logic, a logical formula is associated to each marking and each transition. The left hand of the initial sequent must hold the list of all the transitions that can be fired to obtain a marking $M_{f}$ from an initial marking $M_{0}$. The building of the proof generates a proof tree beginning by a sequent and finishing by the identity axiom. For a given Petri Net, the translation is performed as follows:

- An atomic proposition $P$ is associated with each place $p$ of the Petri Net,

- A single sequent using the multiplicative conjunction TIMES $(\otimes)$, is associated with each marking, pre-condition and post-condition of transition,

- To each transition $t$ of the net an implicative formula is defined as follows:

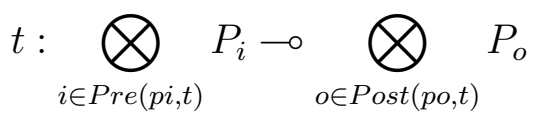

To show reachability between two markings $M_{0}$ and $M_{f}$, the proof of the sequent $M_{0}, t_{1}, \ldots, t_{n} \vdash M_{f}$ is performed as follows: first, the initial marking $M_{0}$ is replaced in separate atoms by applying the $\otimes_{L}$ rule as many times as necessary. Then, by applying ${ }^{\circ} L$, causality relation of atoms (from $M_{0}$ to $M_{f}$ ) can be extracted. Each time $\multimap_{L}$ rule is applied, $\otimes_{L}$ rule is applied if necessary to separate atoms relied by $\otimes$. The proof continues essentially at the right side of the tree because after each application of $\multimap_{L}$ rule, the left member is proven by using, if necessary, the $\otimes_{R}$ rule. The sequent proof ends when all implicative formulae (expressing transitions) are eliminated.

\section{Linear logic and Colored Petri Nets}

The application of linear logic to CPN reachability analysis requiers translation between the two models (from CPN to linear logic). This translation have to respect characteristics of CPN, particularly tokens types and arc expressions which not exist in ordinary PN. To express tokens differentiation and their values evolution in CPNs, the predicative linear logic is very limited ; this is why, in this work, the first order linear logic was preferred. This section presents the 
linear logic fragment used to translate CPNs wich is First Order Multiplicative Intuitionistic Linear Logic (noted MILL1) and then it presents the translation algorithm.

4.1 First Order Multiplicative Intuitionistic Linear Logic

MILL1 language is defined as follow:

Alphabet: The alphabet consists on disjoint sets: a set of variable symbols (e.g. $x, y$ ), a set of function symbols (e.g. $f, g, h$ ), a set of relation symbols (e.g. $R, S, T)$, the binary connectives $(\otimes, \multimap)$ and quantifier $\forall$. Each language $\mathcal{L}$ is equipped with a map from $\mathcal{L}$ to the set of natural integers ar $: \mathcal{L} \rightarrow \mathbb{N}$. This map ar stands for symbol arity.

Terms: Given a language $\mathcal{L}$, the first-order terms over $\mathcal{L}$ are defined by the syntactic category below:

$$
\begin{aligned}
\tau::= & x \\
\mid & f\left(\tau_{1}, \ldots, \tau_{n}\right)
\end{aligned}
$$

where $x$ ranges over the variables and $f$ belongs to the function symbols of $\mathcal{L}$ such that $\operatorname{ar}(f)=n$.

Formulas: First-order formulas (or formulæ) of MILL are defined by the inductive syntactic category below:

$$
\begin{aligned}
\varphi, \phi::= & R\left(\tau_{1}, \ldots, \tau_{n}\right) \\
\mid & \varphi \otimes \phi \\
& \varphi \multimap \phi \\
& \forall x \cdot \varphi
\end{aligned}
$$

where $R$ belongs to the relation symbols of $\mathcal{L}$ such that $\operatorname{ar}(R)=n$ and where the variable $x$ occurrences in formula $\varphi$ are bound in formula $\forall x \cdot \varphi$ by the universal quantifier. Variables that are not bound by a quantifier are called free.

Sequents: If $\Gamma$ is a multiset of formulas separated by "," and $\varphi$ is a formula then $\Gamma \vdash \varphi$ is a sequent. By taking $\Gamma$ as a multiset we will implicitly assume that the sequent comma "," is associative and commutative. $\Gamma$ is called the antecedent of the sequent and $\varphi$ the succedent.

Proofs in MILL1 are given in terms of proof trees that are inference rule composition over judgments. Judgments are pairs of a set of formulas $\Gamma$ and a formula $\varphi$ that are written $\Gamma \vdash \varphi$. This means that the formula $\varphi$ is a logical consequence of the conjunction of those of $\Gamma$. Inference rules ( $n$-ary) are relations between $n+1$ judgments that are noted as follows:

$$
\frac{\Gamma_{1} \vdash \varphi_{1} \quad \cdots \quad \Gamma_{n} \vdash \varphi_{n}}{\Gamma \vdash \varphi}
$$

which means that it is sufficient to establish the below-rule judgment $\Gamma \vdash \varphi$ if the above-rule ones hold; in other words, to establish the below-rule judgment it is necessary to prove the above-rule judgments $\Gamma_{i} \vdash \varphi_{i}(1 \leqslant i \leqslant n)$. Inference rules of MILL are given in figure 2 .

$$
\begin{aligned}
& \frac{\Gamma, \varphi \vdash \phi}{\varphi \vdash \varphi} i d \quad \frac{{ }_{r}}{\Gamma \vdash \varphi \multimap \phi} \\
& \frac{\Gamma \vdash \varphi \quad \phi, \Delta \vdash \psi}{\Gamma, \varphi \multimap \phi, \Delta \vdash \psi} \multimap_{l} \\
& \frac{\Gamma \vdash \varphi \quad \Delta \vdash \phi}{\Gamma, \Delta \vdash \varphi \otimes \phi} \otimes_{r} \quad \frac{\Gamma, \varphi, \phi \vdash \psi}{\Gamma, \varphi \otimes \phi \vdash \psi} \otimes_{l} \\
& \frac{\Gamma \vdash \varphi}{\Gamma \vdash \forall x \cdot \varphi} \forall_{r} \quad(*) \quad \frac{\Gamma, \varphi \vdash \psi}{\Gamma, \forall x \cdot \varphi \vdash \psi} \forall_{l}
\end{aligned}
$$

The constraint $(*)$ requires that the variable $x$ isn't free in formulas of $\Gamma$.

Figure 2: First-Order Multiplicative Intuitionistic Linear Logic

\subsection{Translation of a Colored Petri Net to MILL1}

For a given CPN, the translation is performed as follows:

- A unary atomic predicate $R$ is associated with each place $p$ of the CPN,

- A single sequent using the multiplicative conjunction TIMES $(\otimes)$, is associated with each marking, pre-condition and post-condition of transition,

- To each transition $t$ of the net an implicative formula is defined as follows:

$$
t: \forall x_{1} \ldots \forall x_{i}\left(\bigotimes_{i \in \operatorname{Pre}(p i, t)} R_{i}\left(x_{i}\right) \multimap \bigotimes_{o \in \operatorname{Post}(p o, t)} R_{o}\left(f_{o}\left(X_{o}\right)\right)\right)
$$

Where $x_{i}$ are variables marking the input arcs of $t$. $f_{o}$ are functions associated to the output arcs of $t$. $X_{o}$ are vectors composed of different associations of $x_{i}$.

The following example translates the CPN illustrated in Fig. 3 to its equivalent in MILL1.

- places $p_{i}$ are translated by unary atomic predicates $x \mapsto R_{i}(x)$ where $(1 \leqslant i \leqslant 5)$;

- the transition $t_{1}$ is translated by the formula: $\varphi_{1} \widehat{=} \forall x \cdot\left(R_{1}(x) \multimap R_{2}(f(x)) \otimes R_{4}(g(x))\right)$

- transition $t_{2}$ is translated by the formula: $\varphi_{2} \widehat{=}$ $\forall y \cdot \forall z \cdot\left(R_{2}(y) \otimes R_{5}(z) \multimap R_{3}(h(y, z))\right)$ 


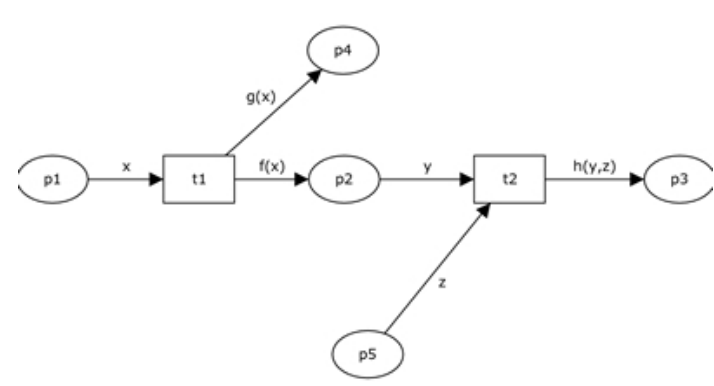

Figure 3: Colored Petri Net Example

- the initial state is translated by the formula: $\phi_{0} \widehat{=}$ $R_{1}(i) \otimes R_{5}(j)$

- the final state is translated by the formula: $\phi \widehat{=}$ $R_{3}(a) \otimes R_{4}(b)$

The remaining of the example treats a case of reachability between two markings $M_{0}$ and $M_{f}$ where: $M_{0}=\langle i\rangle \cdot p 1+\langle j\rangle . p 5$ and $M_{f}=\langle a\rangle \cdot p 3+$ $\langle b\rangle . p 4$. The reachability between $M_{0}$ and $M_{f}$ is obtained by the means of the judgment $\phi_{0}, \varphi_{1}, \varphi_{2} \vdash \phi$ following proof in MILL1 (see proof tree in Fig.4): first, the initial state formula $\phi_{0}$ is treated by $\otimes_{l}$, then the transition $\sigma_{1}$ is treated by $\forall_{l}$ and $\multimap_{l}$. The result of the first transition is treated by $\otimes_{l}$. The second transition $\sigma_{2}$ is treated twice by $\forall_{l}$ and $\multimap$. The results of the second transition is treated by $\otimes_{r}$. In a parallel direction, we treat the final state formula $\phi$ by $\otimes_{r}$ as well. We finally apply $i d$ rules and unify the remaining equations by this substitution $\varsigma$ :

$$
\begin{aligned}
& \varsigma(a)=h(f(i), j) \\
& \varsigma(b)=g(i)
\end{aligned}
$$

\section{Case study}

This example is inspired from (Cho, Hing, and Cha 1996). It describes a shutdown system (SDS2) for a Korean nuclear power plant. SDS2 monitors the state of a nuclear reactor such as its pressure and power and generates a trip signal (shutdown) if the nuclear reactor becomes unsafe (means the pressure is too high or too low). There are several trip parameters in SDS2. Here, is described only PDL trip parameter. Fig. 5 shows a CPN for PDL trip parameter of SDS2.

To control the PDL trip parameter, SDS2 monitors the status of the nuclear reactor such as core differential pressure, $\Delta P$ and log power signal, $\phi_{L O G}$. In Fig.5, the places Pressure and Power represent the current value of $\triangle P$ and $\phi_{L O G}$, respectively. $P 1, P 2$, and $P 3$ represent the states of SDS2, respectively. PDLTrip represents the PDL trip parameter whose value is determined as follows:

- Determine the trip conditioning status: If $\phi_{L O G}<2739 m V$, disable the trip. Otherwise enable the trip.

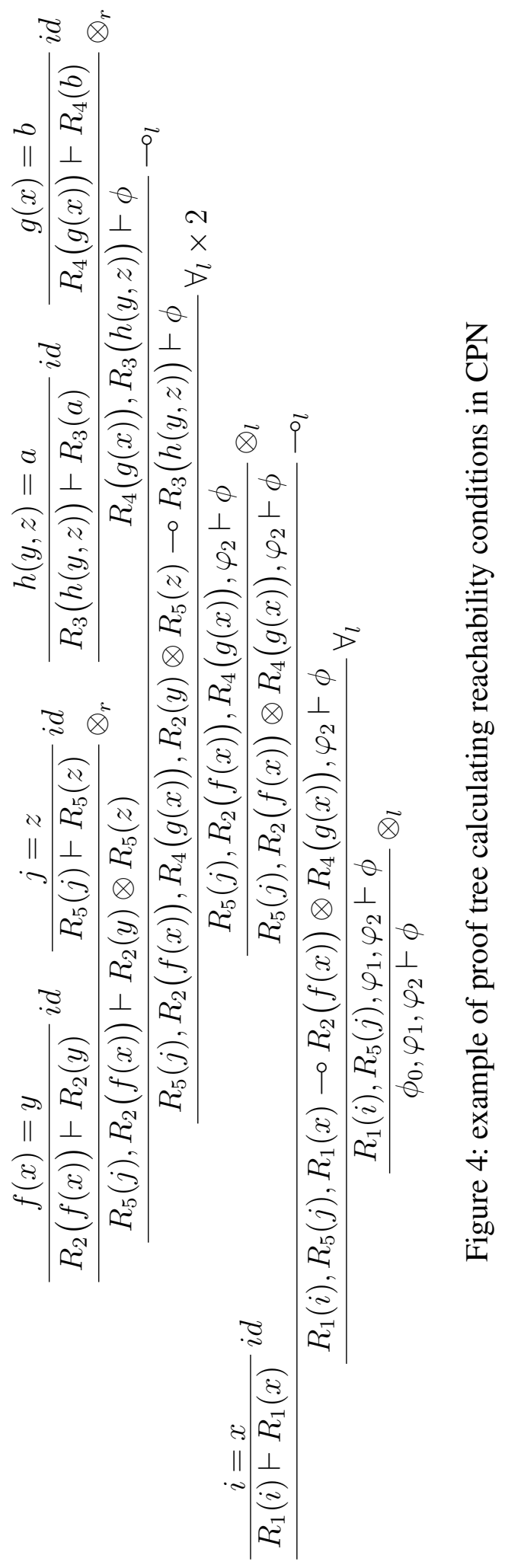




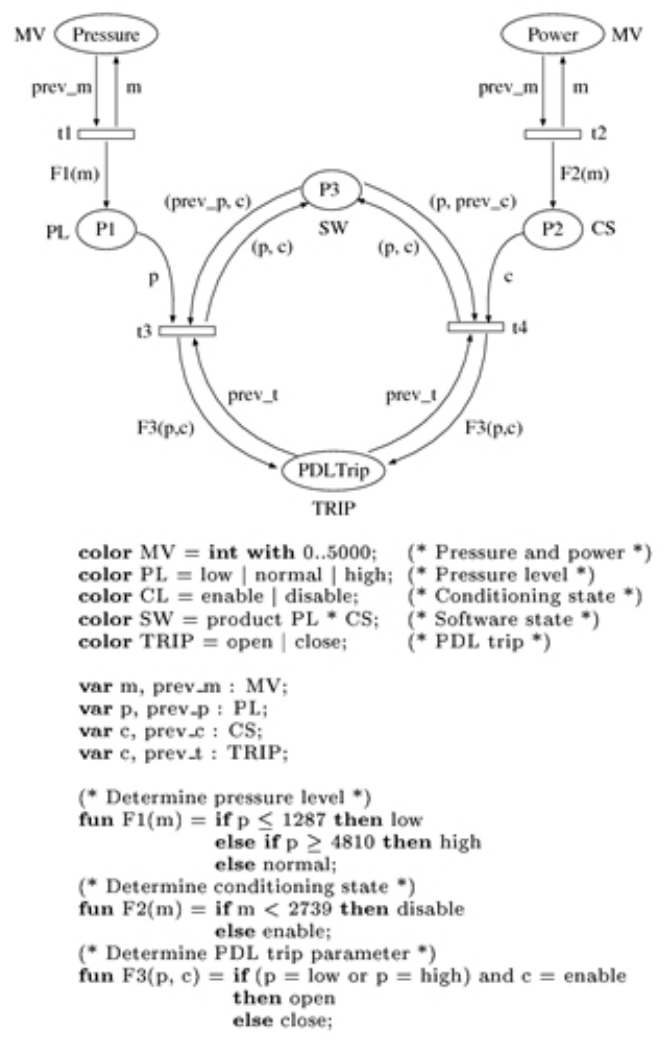

Figure 5: CPN for PDL trip parameter

- Determine the PDL trip parameter: If the trip conditioning is enabled and $(\Delta P<1287 \mathrm{mV}$ or $\Delta P>4810 m V$ ), open PDL trip parameter. Otherwise, close PDL trip parameter.

Let's consider the state vector (Pressure, Power, P1, P2, P3, PDLtrip). The state $(x, y, ?, ?, ?, z)^{1}$ is a failure state if $x \leq 1287$ or $x \geq 4810, y \geq 2739$ and $z=$ close

The CPN inversion can be performed by applying a parallel transformation for transitions $t 1, t 2$ and a parameterized parallel one for $t 3, t 4$ (see Fig.6)

Transitions $t 1$ and $t 2$ : The firing of these transitions corresponds to an update of Pressure and Power tokens values. Old values are deleted and replaced by those of the variable $m$. New token values in $P 1$ and $P 2$ are calculated using functions $F 1$ and $F 2$ respectively. The inversion of these transitions exploits values produced by $F 1$ and $F 2$ to find backward those in Pressure and Power using the functions F1inverse $(p)$ and F2inverse $(c)$.

Transitions $t 3$ and $t 4$ : These transitions are in the same time parallel and parametric. Parallel because the output arcs expressions are functions of same input variables. Parametric cause of the multi variable function $F 3$. The inversion displays guards $[t=$

\footnotetext{
${ }^{1}$ the character "?" stands for a missing information
}

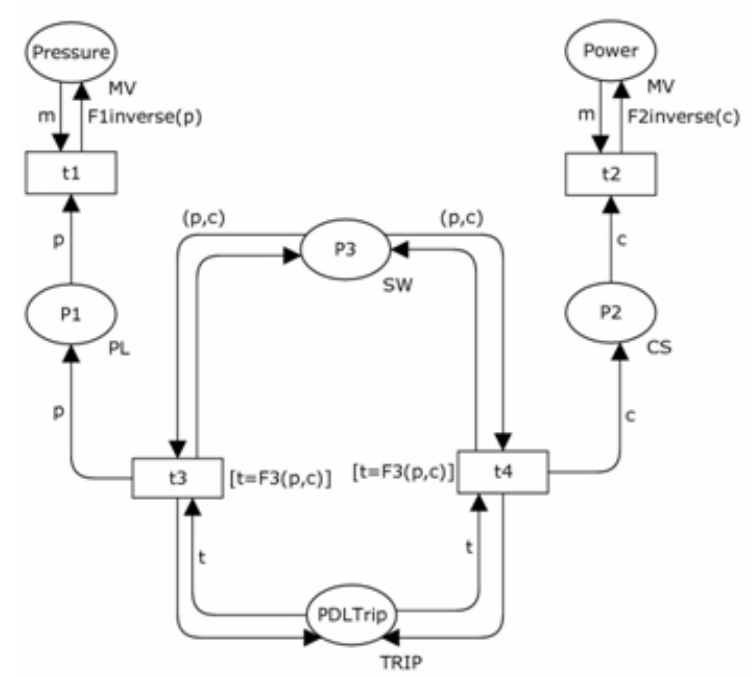

Figure 6: inverse CPN for PDL trip parameter

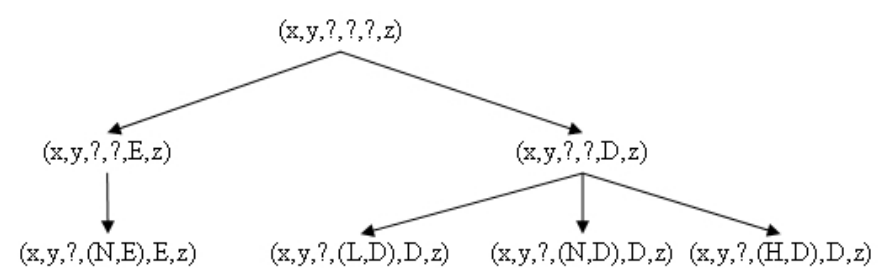

Figure 7: First inverse reachability tree for PDL trip parameter

$F 3(p, c)]$. The inversion of the function $F 3$ is different in $t 3$ and $t 4$. Around $t 3$, the variable $c$ is constant, on the other hand, around $t 4$ the variable $p$ is constant.

Backward analysis: In this paragraph, same results are obtained using both structural backward reachability analysis (inverse CPN) and linear logic (sequent proofs). The structure of the inverse $\mathrm{CPN}$ is translated in MILL1 as described in the section 4. Then proof trees are calculated.

Let's consider, in the inverse CPN, the same state vector as the original CPN: (Pressure, Power, P1, P2, P3, PDLtrip). The failure marking is $(x, y, ?, ?, ?, z)$ where $x \leq 1287$ or $x \geq 4810, y \geq 2739$ and $z=$ close.

The marking enhancement consists in definition of possible values of unknown tokens (in $P 1, P 2, P 3$ ) starting by conditioning state represented by the token value in $P 2$ which corresponds also to the value of the variable $c$ in the schema. The set of possible colors in this case (in P2) is limited, by definition, to two values: Enable and Disable (noted $E$ and $D$ respectively). Corresponding values for the variable $p$ are calculated toward the parameterized inverse of the function $F 3$ which inputs are $z$ and $c$. Results are shown in the tree Fig.7.

Another manner to enhance the marking is to define possible values of unknown tokens (in $P 1, P 2$, P3) starting by Pressure level represented by the to- 


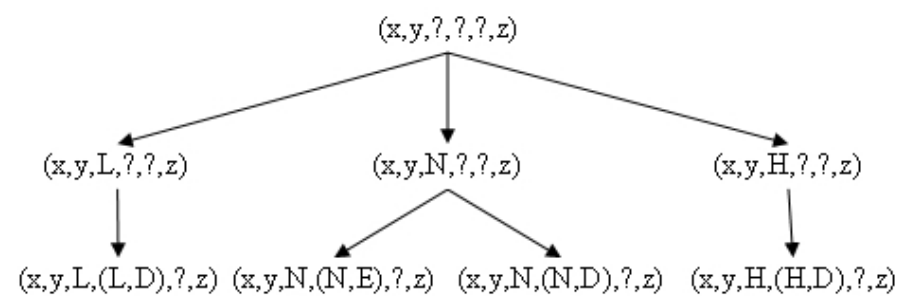

Figure 8: Second inverse reachability tree for PDL trip parameter

ken value in $P 1$ which corresponds also to the value of the variable $p$ in the schema. The set of possible colors in this case (in $P 1$ ) is limited, by definition, to three values: Low, Normal and High (noted L, $N$ and $H$ respectively). Corresponding values for the variable $c$ are calculated toward the parameterized inverse of the function $F 3$ which inputs are $z$ and $p$. Results are shown in the tree Fig.8.

\section{Conclusion}

This paper presents the application of the linear logic (LL) to express reachability, and especially, backward reachability in Colored Petri Nets. To do, the equivalence between LL and Petri Nets is exploited. The LL fragment used is the Multiplicative Intuitionistic Linear Logic (MILL). This equivalence is extended to CPN. The conversion between CPN and MILL must respect properties of $\mathrm{CPN}$, i.e. tokens are from of a certain type and they are transformed using arcs expressions. The LL fragment in which these constraints can be expressed is the MILL1. The reachability in CPN is expressed in LL by sequents which can be proven and proof trees can be constructed.

The main application field for this work is the formal model diagnostics during the conception. The system initial state is usually well determined and so is the final state which in this case represents an undesired state. The proposed method can provide answers to the possibility of undesired event occurrence, the earliest failure time, the system evolution vector, etc.

The perspectives start with the development of an automated tool for a verification of the CPN structure in order to be acceptable for the backward reachability analysis. The theory advances include the generalization of transformation rules to composed functions. The second path to explore is the possible of presence of non deterministic time constraints for the transition firing. The study of stochastic firing vectors is also considered as a potential subject of interest.

\section{REFERENCES}

Cho, S. M., H. S. Hing, and S. D. Cha (1996). Safety analysis using colored petri nets. In Proc. Asia-Pacific Software Engineering Conference (APSEC-96), 4-7 December 1996, Seoul, Korea, pp. 176-183.
F.Girault (1997). Formalisation en Logique Linaire du fonctionnement des rseaux de Petri. Ph. D. thesis, Universit Paul Sabatier, Toulouse.

G.Gentzen (1969). The Collected Works of Gerhard Gentzen. Amsterdam: North-Holland Publishing Company.

Girard, J. (1987). Linear logic. Theorical Computer Sciences 50, 1-102.

H.Demmou, S.Khalfaoui, E.Guilhem, and R.Valette (2004). Critical scenarios derivation methodology for mechatronic systems. $R e$ liability Engineering and System Safety 84, 33-44.

Jensen, K. and G. Rozenberg (1991). High-level Petri Nets : Theory and Application. Germany: Springer-Verlag.

Khalfaoui, S. (2003, Septembre). Mthode de recherche des scnarios redouts pour l'valuation de la sret de fonctionnement des systmes mcatroniques du monde automobile. $\mathrm{Ph}$. D. thesis, Institut National polytechnique de Toulouse (France).

Lincoln, P. (1992). Linear logic. SIGACT News 23(2), 29-37.

M.Bouali, P.Barger, and W.Schon (2009a, June). Colored petri nets inversion for backward reachability analysis. Second IFAC Workshop on Depandable Control of Descrete Systems (DCDS'09), Bari, Italia.

M.Bouali, P.Barger, and W.Schon (2009b, May). Towards an efficient structural analysis of colored petri nets. International Conference on Industrial Engineering and Systems Management (IESM'09), Montreal, Canada.

Metropolis, N. and S. Ulam (1949, September). The monte carlo method. Journal of American Statistical Association 44(247), 335-341.

Petri, C. A. (1962). Communication with automata. Ph. D. thesis, Darmstadt Institut fr Instrumentelle Mathematik, Bonn (Germany). 